\title{
Adiabatic electronic motion in forming covalent bond
}

\author{
Michihiro Okuyama* \\ KONICA MINOLTA, Inc., Address, 2970 Ishikawa-machi, Hachioji-shi Tokyo 192-8505, Japan \\ Fumihiko Sakata \\ Institute of Applied Beam Science, Graduate School of Science and Technology, \\ Ibaraki University, Address, Mito 310-8512, Japan
}

(Dated: October 18, 2018)

\begin{abstract}
In studying a dynamical process of the chemical reaction, it is decisive to get appropriate information from an electronic current density. To this end, we divide one-body electronic density into a couple of densities, that is, an electronic sharing density and an electronic contraction density. Since the one-body electronic current density defined directly through the microscopic electronic wave function gives null value under the Born-Oppenheimer molecular dynamics, we propose to employ the Maxwell's displacement current density defined by means of the one-body electronic density obtained under the same approximation. Applying the electronic sharing and the electronic contraction current densities to a hydrogen molecule, we show these densities give important physical quantities for analyzing a dynamical process of the covalent bond.
\end{abstract}

\section{INTRODUCTION}

One of the basic problems in the contemporary chemical reaction theory is to explore and to properly describe how a complex molecular system composed of many electrons and nuclei splits into many parts, and how some of these parts recombine with each other under various external situations. For this aim, there have been many works based on the Born-Oppenheimer approximation (BOA) [1] or Ehrenfest's theorem to establish an appropriate description on a relation between macroscopic and microscopic dynamics, classical and quantum dynamics, and light particle fast motion and heavy particle slow motion, and so forth [2-11]. In recent studies on these subjects, electronic motion caused by nuclear motion in molecules has been studied [12-20], and it is generally being accepted such an important view point that a chemical bond process and a bond dissociation process are induced not by all electrons of a system but only by a part them. In order to understand the chemical reaction mechanism, therefore, it is decisive to explore the electronic motion taking place in association with a reactants-to-product process, and to examine the electronic current density which tells us a direction of electron flow and a number of participating electrons in the process. Aiming at studying the electronic current density, one has to exploit the physical quantities with vector character. Since the physical quantities used in the above mentioned works have scalar character, it is not suitable to employ them for studying the electronic current density. Here, it is meaningful to refer to some works [21, 22], which explored not on the vector type electronic current density generated by the adiabatic nuclear motion, but on that generated by the non-adiabatic effects.

\footnotetext{
* michihiro.okuyama@konicaminolta.com michihiro.okuyama@gmail.com
}

The aim of this paper is to introduce a new quantity called an electronic sharing current density (ESCD) and to show its usefulness in studying the chemical reaction mechanism. In order to define ESCD, one needs the onebody electronic current density (OECD) which is usually given by the probability current density as

$$
\mathbf{j}(\mathbf{r}, t)=\frac{1}{2 i}\left(\psi^{*}(\mathbf{r}, t) \nabla \psi(\mathbf{r}, t)-\psi(\mathbf{r}, t) \nabla \psi^{*}(\mathbf{r}, t)\right),
$$

in unit of the atomic unit (a.u.) [23]. Here $\psi(\mathbf{r}, t)$ denotes an electronic wave function of the system. When the electronic motion is described by the Born-Oppenheimer molecular dynamics (BOMD) [24], there holds $\mathbf{j}(\mathbf{r}, t)=\mathbf{0}$ because of real-valuedness of the electronic wave function $\psi(\mathbf{r}, t)$. Since the same difficulty also arises in an electronic motion accompanying a quantum nuclear motion within BOA [25-27], there have been many studies to overcome this difficulty [28-36]. Although there are some works $[28,33]$ which try to describe a flow of the electronic density within BOMD, physical quantities adopted in these works do not correctly express OECD because they are not able to describe such an electronic current that flows out from the one-body electronic density (OED) at every point of a space.

In this paper, we treat time-dependence of OED in a way consistent with the basic assumption of BOMD, instead of describing the electronic current density in terms of the microscopic wave function. That is, the time dependence of OED pertaining to the chemical bond and bond dissociation processes between atoms within a molecular system is described by classical relative motions between nuclei. Namely, we define OECD by applying a continuity relation of the classical electrodynamics to OED, whose time dependence comes through a set of nuclear coordinates. OECD thus introduced describes a classical or macroscopic electronic motion accompanying the classical nuclear motion which expresses the chemical bond and the bond dissociation processes among the atoms in the molecular system. OECD is then divided 
into two terms. One is composed of a set of overlap distributions [37] between atomic orbitals belonging to different atoms, and is called ESCD, whereas the other consists of those belonging to the same atom, and is called an electronic contraction current density (ECCD).

Showing a usefulness of a pair of new quantities discussed just the above, we focus on the electronic motion in the covalent bond formation process in the hydrogen molecule. Many studies on the covalent bond have been done by using simple systems $\mathrm{H}_{2}^{+}$and/or $\mathrm{H}_{2}$ within BOA [37-46], and there have been discussed that 1) electronic delocalization and 2) orbital contraction plays decisive roles in the bond formation process [37, 45]. By applying two new quantities to the covalent bond formation process in the hydrogen molecule, we show how well the electronic motion accompanying the (classical) nuclear motion is described by the present macroscopic description. It is also shown that there occurs an increase of an electronic energy in the individual hydrogen atoms in compensation with an increase of depth in the potential between two hydrogen nuclei, which favors the covalent bond in forming the hydrogen molecule. Although the microscopic quantum effects of the electronic current density beyond the adiabatic treatment are not considered in the present paper, we discuss them in a future publication.

This paper is organized as follows: Dividing OED into a sharing and a contraction parts, and using the Maxwell's displacement current density, we discuss a theoretical basis for analyzing the chemical reaction in Sect. II. In order to explore how the covalent bond is formed, in Sect. III, we also divide the electronic Hamiltonian into many parts which play respective roles in forming the covalent bond. The computational details and numerical results of our treatment applied for $\mathrm{H}_{2}$ system are discussed in Sect. IV and summary is given in Sect. V.

\section{ELECTRONIC CURRENT DENSITY EXPRESSED BY MAXWELL'S DISPLACEMENT CURRENT DENSITY}

We consider the system whose number of nuclei and electrons are $N_{n}$ and $N_{e}$, respectively. In BOMD, the equations of motion for nuclei and electrons are expressed as [24]

$$
\begin{aligned}
\hat{H}_{e}(\{\mathbf{R}(t)\}) \varphi_{e}(\{\mathbf{r}\} ;\{\mathbf{R}(t)\}) & =E_{e}(\{\mathbf{R}(t)\}) \varphi_{e}(\{\mathbf{r}\} ;\{\mathbf{R}(t)\}), \\
M_{I} \ddot{\mathbf{R}}_{I} & =-\nabla_{I} E_{e}(\{\mathbf{R}(t)\}),
\end{aligned}
$$

where, $\{\mathbf{r}\}$ and $\{\mathbf{R}(t)\}$ collectively express a set of electronic and nuclear position coordinates. $\hat{H}_{e}(\{\mathbf{R}(t)\})$ is an electronic Hamiltonian, and $E_{e}(\{\mathbf{R}(t)\})$ and $\varphi_{e}(\{\mathbf{r}\} ;\{\mathbf{R}(t)\})$ are an eigen energy and an eigen state of the electronic Hamiltonian, and a dot symbol · denotes a time derivative. $M_{I}, \mathbf{R}_{I}$ and $\nabla_{I}$ express a mass, a position coordinate and its gradient of the $I$-th nucleus. In the restricted Hartree-Fock (RHF) approximation [47], the eigen state is expressed in terms of a single Slater determinant wave function composed by a set of spin orbitals $\left\{\phi_{i}\left(\mathbf{r}_{i} ;\{\mathbf{R}(t)\}\right)|\alpha\rangle, \phi_{i}\left(\mathbf{r}_{i} ;\{\mathbf{R}(t)\}\right)|\beta\rangle ; i=\right.$ $\left.1, \cdots, N_{e} / 2\right\}$ where $\phi_{i}\left(\mathbf{r}_{i} ;\{\mathbf{R}(t)\}\right)$ and $|\alpha, \beta\rangle$ denote a set of spacial orbitals and of spin functions, respectively.

In order to define OECD within BOMD, one may rewrite the right-hand side of Eq. (3) as [48],

$$
\begin{array}{r}
-\nabla_{I} E_{e}(\{\mathbf{R}(t)\})=-Z_{I} \int d \mathbf{r} \frac{\mathbf{R}_{I}(t)-\mathbf{r}}{\left|\mathbf{R}_{I}(t)-\mathbf{r}\right|^{3}} \rho_{e}(\mathbf{r} ;\{\mathbf{R}(t)\}) \\
+Z_{I} \int d \mathbf{R} \frac{\mathbf{R}_{I}(t)-\mathbf{R}}{\left|\mathbf{R}_{I}(t)-\mathbf{R}\right|^{3}} \rho_{n}(\mathbf{R} ;\{\mathbf{R}(t)\}), \quad(4)
\end{array}
$$

where

$$
\begin{aligned}
\rho_{e}(\mathbf{r} ;\{\mathbf{R}(t)\}) & =N_{e} \int d \mathbf{r}_{2} \cdots d \mathbf{r}_{N_{e}}\left|\varphi_{e}(\{\mathbf{r}\} ;\{\mathbf{R}(t)\})\right|^{2} \\
& =2 \sum_{i=1}^{N_{e} / 2}\left|\phi_{i}(\mathbf{r} ;\{\mathbf{R}(t)\})\right|^{2} \\
\rho_{n}(\mathbf{R} ;\{\mathbf{R}(t)\}) & =\sum_{I=1}^{N_{n}} Z_{I} \delta\left(\mathbf{R}-\mathbf{R}_{I}(t)\right)
\end{aligned}
$$

In Eqs. (5) and (6), $\mathbf{r}$ and $\mathbf{R}$ mean position coordinates of a single electron and a single nucleus, and $Z_{I}$ denotes an atomic number of the $I$-th nucleus. Although two integrals in the right-hand side of Eq. (4) express electric fields at $\{\mathbf{R}(t)\}$ generated by the electronic and nuclear charge distributions, it should be emphasized that each of them is derived from different theoretical frameworks. Namely, the electronic charge distribution $\rho_{e}(\mathbf{r} ;\{\mathbf{R}(t)\})$ is determined by the quantum mechanics, whereas the nuclear charge distribution $\rho_{n}(\mathbf{R} ;\{\mathbf{R}(t)\})$ is given by the $N_{n}$ point charges $Z_{I}$ located at the position determined by the classical Newtonian equation in Eq. (3).

In order to derive the continuity equation for OED, let us introduce an electric field $\left.\mathbf{F}_{e}(\mathbf{x} ;\{\mathbf{R}(t)\})\right)$ by means of the first term in the right-hand side of Eq. (4) as

$$
\left.\mathbf{F}_{e}(\mathbf{x} ;\{\mathbf{R}(t)\})\right)=-\int d \mathbf{r} \frac{\mathbf{x}-\mathbf{r}}{|\mathbf{x}-\mathbf{r}|^{3}} \rho_{e}(\mathbf{r} ;\{\mathbf{R}(t)\}),
$$

where $\mathbf{x}$ denotes a spacial parameter. Equation (7) satisfies the following electrostatic relations

$$
\begin{gathered}
\nabla_{\mathbf{x}} \cdot \mathbf{F}_{e}(\mathbf{x} ;\{\mathbf{R}(t)\})=-4 \pi \rho_{e}(\mathbf{x} ;\{\mathbf{R}(t)\}), \\
\nabla_{\mathbf{x}} \times \mathbf{F}_{e}(\mathbf{x} ;\{\mathbf{R}(t)\})=\mathbf{0} .
\end{gathered}
$$

Taking a time derivative of Eq. (8), one gets a continuity equation

$$
\frac{\partial}{\partial t} \rho_{e}(\mathbf{x} ;\{\mathbf{R}(t)\})+\nabla_{\mathbf{x}} \cdot\left(\frac{1}{4 \pi} \frac{\partial}{\partial t} \mathbf{F}_{e}(\mathbf{x} ;\{\mathbf{R}(t)\})\right)=0 .
$$

From the above equation, OECD is defined as

$$
\mathbf{j}_{e}(\mathbf{x} ;\{\mathbf{R}(t)\},\{\dot{\mathbf{R}}(t)\})=\frac{1}{4 \pi} \frac{\partial}{\partial t} \mathbf{F}_{e}(\mathbf{x} ;\{\mathbf{R}(t)\}),
$$


where $\{\dot{\mathbf{R}}(t)\}$ denotes a set of velocity vectors of all nuclei. Equation (11) is the Maxwell's displacement current density in the classical electrodynamics [49]. Within BOMD, each vector component $j_{e i}(\mathbf{x} ;\{\mathbf{R}(t)\},\{\dot{\mathbf{R}}(t)\})$ with $i=x, y, z$ is given as

$$
j_{e i}(\mathbf{x} ;\{\mathbf{R}(t)\},\{\dot{\mathbf{R}}(t)\})=\frac{1}{4 \pi} \sum_{I=1}^{N_{n}} \dot{\mathbf{R}}_{I} \cdot \nabla_{I} F_{e i}(\mathbf{x} ;\{\mathbf{R}(t)\}),
$$

where $F_{e i}(\mathbf{x} ;\{\mathbf{R}(t)\})$ means $i=x, y, z$-component of the electric field in Eq. (7). As is clear from Eq. (12), the displacement current density depends not only on the nuclear position coordinates but also on the nuclear velocities. Therefore, when the velocities of all nuclei are null vectors, the displacement current density in Eq. (12) is also the case. Here, it should be noticed that Eq. (12) is compatible with the basic assumption of BOMD where the time development of the electronic state is postulated to accompany the nuclear motion.

To explore the dynamical process of forming the covalent bond, let us divide OED into a couple of densities as

$$
\begin{aligned}
\rho_{e}(\mathbf{r} ;\{\mathbf{R}(t)\}) & =\sum_{I, J=1}^{N_{n}} \sum_{\mu(I)}^{\mathrm{A} O} \sum_{\nu(J)}^{\mathrm{A} O} \rho_{\mu(I) \nu(J)}(\{\mathbf{R}(t)\}) \\
& \times \chi_{\mu(I)}(\mathbf{r}) \chi_{\nu(J)}(\mathbf{r}) \\
= & \rho_{e}^{s}(\mathbf{r} ;\{\mathbf{R}(t)\})+\rho_{e}^{c}(\mathbf{r} ;\{\mathbf{R}(t)\}),
\end{aligned}
$$

where

$$
\begin{aligned}
\rho_{e}^{s}(\mathbf{r} ;\{\mathbf{R}(t)\}) & =\sum_{I \neq J}^{N_{n}} \sum_{\mu(I)}^{\mathrm{A} O} \sum_{\nu(J)}^{\mathrm{A} O} \rho_{\mu(I) \nu(J)}(\{\mathbf{R}(t)\}) \\
\times \chi_{\mu(I)}(\mathbf{r}) \chi_{\nu(J)}(\mathbf{r}), & \\
\rho_{e}^{c}(\mathbf{r} ;\{\mathbf{R}(t)\}) & =\sum_{I=1}^{N_{n}} \rho_{e}^{c I}(\mathbf{r} ;\{\mathbf{R}(t)\}),
\end{aligned}
$$

with

$$
\begin{aligned}
\rho_{e}^{c I}(\mathbf{r} ;\{\mathbf{R}(t)\})=\sum_{\mu(I), \nu(I)}^{\mathrm{A} O} \rho_{\mu(I) \nu(I)}(\{\mathbf{R}(t)\}) \\
\times \chi_{\mu(I)}(\mathbf{r}) \chi_{\nu(I)}(\mathbf{r}) .
\end{aligned}
$$

Here, $\chi_{\mu(I)}(\mathbf{r})$ is an atomic orbital $(\mathrm{AO})$ of the $I$-th nucleus located at the position $\mathbf{R}_{I}(t)$ in a state characterized by the quantum number $\mu(I)$, and a summation symbol on $\mu(I)$ means a sum over the AOs at $\mathbf{R}_{I}(t)$. The single-electron wave function $\phi_{i}(\mathbf{r} ;\{\mathbf{R}(t)\})$ determined by RHF equation is expanded by the $\mathrm{AO}$ as

$$
\phi_{i}(\mathbf{r} ;\{\mathbf{R}(t)\})=\sum_{I=1}^{N_{n}} \sum_{\mu(I)}^{A O} c_{\mu(I)}^{i}(\{\mathbf{R}(t)\}) \chi_{\mu(I)}(\mathbf{r}) .
$$

Substituting Eq. (17) into Eq. (5), one may get Eq. (13) where $\rho_{\mu(I) \nu(J)}(\{\mathbf{R}(t)\})$ is a bond order matrix [37] given by

$$
\rho_{\mu(I) \nu(J)}(\{\mathbf{R}(t)\})=2 \sum_{i=1}^{N_{e} / 2} c_{\mu(I)}^{i}(\{\mathbf{R}(t)\}) c_{\nu(J)}^{i}(\{\mathbf{R}(t)\}) .
$$

A partial electronic density $\rho_{e}^{s}(\mathbf{r} ;\{\mathbf{R}(t)\})$ describes a delocalized density distributed over the multiple atoms in a molecule, which is recognized by a conditional summation with $I \neq J$ in Eq. (14), and is hereafter called an electronic sharing density (ESD). On the other hand, $\rho_{e}^{c I}(\mathbf{r} ;\{\mathbf{R}(t)\})$ describes a localized density distributed around the nucleus $I$, and its summation over $I$ given as $\rho_{e}^{c}(\mathbf{r} ;\{\mathbf{R}(t)\})$ in Eq. (15) is called an electronic contraction density (ECD). Here, it should be noted from Eq. (13) that neither of these densities satisfies the continuity equation. By means of Eq. (13), OECD in Eq. (11) is expressed as

$$
\begin{aligned}
& \mathbf{j}_{e}(\mathbf{x} ;\{\mathbf{R}(t)\},\{\dot{\mathbf{R}}(t)\})= \\
& \quad \mathbf{j}_{e}^{s}(\mathbf{x} ;\{\mathbf{R}(t)\},\{\dot{\mathbf{R}}(t)\})+\mathbf{j}_{e}^{c}(\mathbf{x} ;\{\mathbf{R}(t)\},\{\dot{\mathbf{R}}(t)\}),
\end{aligned}
$$

where

$$
\begin{aligned}
\mathbf{j}_{e}^{i}(\mathbf{x} ; & \{\mathbf{R}(t)\},\{\dot{\mathbf{R}}(t)\})=\frac{1}{4 \pi} \frac{\partial}{\partial t} \mathbf{F}_{e}^{i}(\mathbf{x} ;\{\mathbf{R}(t)\}) \\
= & \frac{-1}{4 \pi} \int d \mathbf{r} \frac{\mathbf{x}-\mathbf{r}}{|\mathbf{x}-\mathbf{r}|^{3}} \frac{\partial}{\partial t} \rho_{e}^{i}(\mathbf{r} ;\{\mathbf{R}(t)\}) ; i=s, c .
\end{aligned}
$$

From Eq. (20), it is clear that $\mathbf{j}_{e}^{i}(\mathbf{x} ;\{\mathbf{R}(t)\},\{\dot{\mathbf{R}}(t)\})$ expresses an electronic current density for $\rho_{e}^{i}(\mathbf{r} ;\{\mathbf{R}(t)\})$. Thus, we hereafter call $\mathbf{j}_{e}^{s}(\mathbf{x} ;\{\mathbf{R}(t)\},\{\dot{\mathbf{R}}(t)\})$ and $\mathbf{j}_{e}^{c}(\mathbf{x} ;\{\mathbf{R}(t)\},\{\dot{\mathbf{R}}(t)\})$ ESCD and ECCD, respectively. Using ESCD and ECCD, we examine the dynamical process of forming the covalent bond in the hydrogen molecule.

\section{ENERGY ANALYSIS OF COVALENT BOND}

From the discussions in the previous section, it may be clear that ESD plays a role to form the covalent bond, whereas ECD tries to retain an individuality of each atom. Here, it should be borne in mind that the electronic Hamiltonian has been divided into various parts in order to study how the covalent bond is formed dynamically $[37,41,45]$. Since one may get many important information on the covalent bond by exploring what role is played by each part of the electronic Hamiltonian when the covalent bond is formed, let us first introduce 
an atomic internal energy defined as

$$
\begin{aligned}
& E^{\text {cont }}(\{\mathbf{R}(t)\})=T_{e}^{\text {cont }}(\{\mathbf{R}(t)\})+V_{e n}^{\text {cont }}(\{\mathbf{R}(t)\}), \\
& T_{e}^{\text {cont }}(\{\mathbf{R}(t)\})=\sum_{I=1}^{N_{n}} \sum_{\mu(I) \nu(I)}^{A O} t_{e \mu(I) \nu(I)} \rho_{\mu(I) \nu(I)}(\{\mathbf{R}(t)\}), \\
& V_{e n}^{\text {cont }}(\{\mathbf{R}(t)\})=\sum_{I=1}^{N_{n}} \sum_{\mu(I) \nu(I)}^{A O} v_{e n \mu(I) \nu(I)}^{I} \rho_{\mu(I) \nu(I)}(\{\mathbf{R}(t)\}),
\end{aligned}
$$

which measures how much energy is used to preserve an individuality of each atom. In Eqs. (22) and (23), the following notations are used,

$$
\begin{aligned}
t_{e \mu(I) \nu(I)} & =\int d \mathbf{r} \chi_{\mu(I)}(\mathbf{r}) \frac{-1}{2} \Delta_{\mathbf{r}} \chi_{\nu(I)}(\mathbf{r}), \\
v_{e n \mu(I) \nu(I)}^{I} & =\int d \mathbf{r} \chi_{\mu(I)}(\mathbf{r}) \frac{-1}{\left|\mathbf{r}-\mathbf{R}_{I}(t)\right|} \chi_{\nu(I)}(\mathbf{r}) .
\end{aligned}
$$

By using the atomic internal energy $E^{\text {cont }}(\{\mathbf{R}(t)\})$, the adiabatic potential $E_{e}(\{\mathbf{R}(t)\})$ for nuclear motion defined in Eq. (3) is divided into

$$
\begin{aligned}
& E_{e}(\{\mathbf{R}(t)\})=E^{\text {cont }}(\{\mathbf{R}(t)\})+T_{e}^{\text {shar }}(\{\mathbf{R}(t)\}) \\
& +V_{e n}^{\text {shar }}(\{\mathbf{R}(t)\})+\left\langle\hat{V}_{e e}\right\rangle(\{\mathbf{R}(t)\})+V_{n n}(\{\mathbf{R}(t)\}),
\end{aligned}
$$

where

$$
\begin{aligned}
& T_{e}^{\text {shar }}(\{\mathbf{R}(t)\})=\left\langle\hat{T}_{e}\right\rangle(\{\mathbf{R}(t)\})-T_{e}^{\text {cont }}(\{\mathbf{R}(t)\}), \\
& V_{\text {en }}^{\text {shar }}(\{\mathbf{R}(t)\})=\left\langle\hat{V}_{\text {en }}\right\rangle(\{\mathbf{R}(t)\})-V_{\text {en }}^{\text {cont }}(\{\mathbf{R}(t)\}),
\end{aligned}
$$

with

$$
\begin{array}{r}
\langle\hat{O}\rangle(\{\mathbf{R}\}) \equiv \int d \mathbf{r}_{1} \cdots d \mathbf{r}_{N_{e}} \varphi_{e}^{*}(\{\mathbf{r}\} ;\{\mathbf{R}\}) \hat{O} \varphi_{e}(\{\mathbf{r}\} ;\{\mathbf{R}\}) \\
\hat{O}=\hat{T}_{e}, \hat{V}_{e n}, \hat{V}_{e e} \\
\hat{T}_{e}=\sum_{i=1}^{N_{e}}-\frac{1}{2} \Delta_{\mathbf{r}_{i}}, \quad \hat{V}_{e n}(\{\mathbf{R}\})=\sum_{i=1}^{N_{e}} \sum_{I=1}^{N_{n}} \frac{-Z_{I}}{\left|\mathbf{r}_{i}-\mathbf{R}_{I}\right|}
\end{array}
$$$$
\hat{V}_{e e}=\frac{1}{2} \sum_{i \neq j}^{N_{e}} \frac{1}{\left|\mathbf{r}_{i}-\mathbf{r}_{j}\right|}, V_{n n}(\{\mathbf{R}\})=\frac{1}{2} \sum_{I \neq J}^{N_{n}} \frac{Z_{I} Z_{J}}{\left|\mathbf{R}_{I}-\mathbf{R}_{J}\right|} .
$$

Here, it should be mentioned that $T_{e}^{\text {shar }}(\{\mathbf{R}(t)\})$ and $V_{\text {en }}^{\text {shar }}(\{\mathbf{R}(t)\})$ play a role to characterize electrons shared by multiple atoms. The rest terms in Eq. (26) describe the repulsive Coulomb interactions between arbitrary two electrons $\left\langle\hat{V}_{e e}\right\rangle(\{\mathbf{R}(t)\})$ and between arbitrary two nuclei $V_{n n}(\{\mathbf{R}(t)\})$ which resist the covalent bond.

\section{NUMERICAL RESULTS}

The nuclear motion in the hydrogen molecule is determined by solving Eq. (3) under the following initial condition: At the initial time, two nuclei (two protons) are

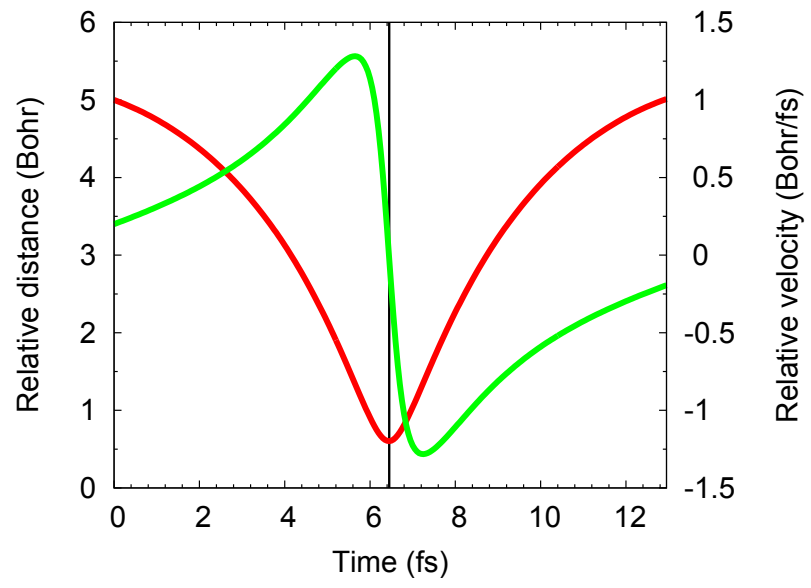

FIG. 1. Time dependence of relative distance $R_{2 z}(t)-R_{1 z}(t)$ (red line) and its velocity $\dot{R}_{2 z}(t)-\dot{R}_{1 z}(t)$ (green line). Units are fs for time, Bohr for relative distance (left vertical axis) and Bohr/fs for relative velocity (right vertical axis).

set on the $z$ axis at \pm 2.5 Bohr separated by 5 Bohr. Two nuclei are boosted with relative velocity 0.2 Hartree/fs along the $z$ axis.

We calculate the forces acting on the nuclei in Eq. (3) by means of RHF method with the STO-3G basis set, and integrated it over 12.95 fs by using the fifth-order Gear method with a time step 0.05 fs. We solve RHF equation by using the GAMESS package [50]. The maximum error in the total energy

$$
E^{t o t}=\sum_{I=1}^{N_{n}} \frac{1}{2} M_{I} \dot{\mathbf{R}}_{I}^{2}+E_{e}(\{\mathbf{R}(t)\})
$$

during the integration is $2.2 \times 10^{-6}$ Hartree which makes us be sure that the error are sufficiently small.

Using thus obtained classical nuclear position, velocity and quantum electronic states, we calculate $\mathbf{j}_{e}(\mathbf{x} ;\{\mathbf{R}(t)\},\{\dot{\mathbf{R}}(t)\}), \quad \mathbf{j}_{e}^{s}(\mathbf{x} ;\{\mathbf{R}(t)\},\{\dot{\mathbf{R}}(t)\}) \quad$ and $\mathbf{j}_{e}^{c}(\mathbf{x} ;\{\mathbf{R}(t)\},\{\dot{\mathbf{R}}(t)\})$.

\section{A. Classical Nuclear Trajectory}

The time dependence of a relative distance as well as that of its velocity between two nuclei in the hydrogen molecule calculated under BOMD are shown in Fig. 1. Since the relative distance is symmetric whereas its velocity is asymmetric with respect to a vertical axis at $t=6.45 \mathrm{fs}$ as illustrated in Fig. 1, the process under consideration expresses a head-on collision of two nuclei in the hydrogen molecule. Therefore, we hereafter focus on it from the initial time till 6.45 fs. 


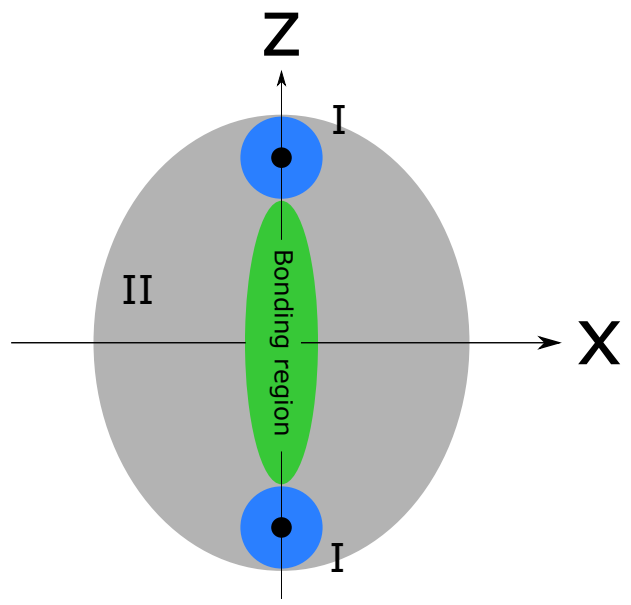

FIG. 2. Schematic division of reaction area into Region I (blue), Region II (gray) and bonding region (green). Two nuclei are indicated by $\bullet$.

\section{B. Electronic Density Dynamics}

Snapshots of OED and OECD are depicted in Fig. 3 which indicates an electronic motion accompanying the classical nuclear trajectory. A couple of small disks localized at two separated nuclei in Fig. 3a.1 exhibit an initial OED. With the passage of time, these disks approach with each other by keeping their shape [Figs. 3a.2, 3a.3], and then fuse to form one molecule [Fig. 3a.4]. In order to explore which parts of OED are activated and how OECD flows when a reaction process proceeds, let us focus our attention on OECD. From Figs. 3b.1-3b.4, it is clearly seen that a relatively large electronic current is generated in two regions: Region I located in the vicinity of each nucleus [Figs. 3b.1-3b.3], and Region II in areas spreading to a left and right sides of the "bonding region" which consists of the molecular axis and its vicinity [Figs. 3b.2, 3b.3]. Namely, a reaction area splits into three characteristic regions schematically shown in Fig. 2. It is also seen that the magnitude of OECD in these region increases with time. Main direction of OECD in Region $\mathrm{I}$ is the same as that of the nuclear motion [Figs. 3c.1-3c.3], whereas that in Region II are directed parallel to the $x$ axis and are headed toward the molecular axis [Figs. 3c.2, 3c.3]. Although the magnitude of OECD shown here increases till $5.8 \mathrm{fs}$, it starts to reduce at 5.8 fs and takes zero at 6.45 fs [Figs. 3b.4, 3c.4], in accordance with the nuclear relative velocity which starts to decrease at 5.8 fs and takes null value at 6.45 fs [Fig. 1].

In the above analysis, it is not clear how the covalent bond emerges out of the initial electronic density in Fig. 3a.1 which expresses electronic density of two isolated hydrogen atoms. Let us discuss ESD and ESCD of which snapshots are shown in Fig. 4. At the initial time, there are almost no distribution of ESD in the $x z$ plane ex-

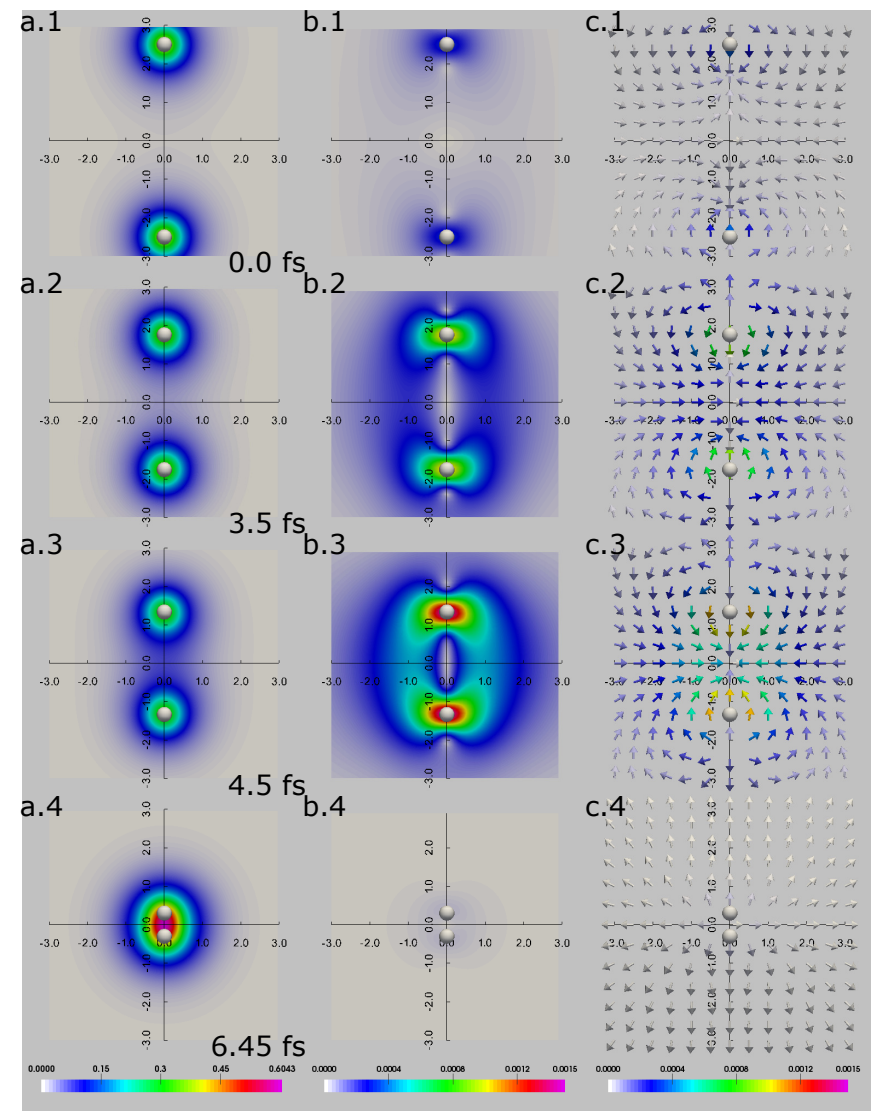

FIG. 3. Color coded contours. a.1-a.4 (left panel): OED $\rho_{e}(x, y, z ;\{\mathbf{R}(t)\})$, b.1-b.4 (center panel): absolute value of OECD $\left|\mathbf{j}_{e}(x, y, z ;\{\mathbf{R}(t)\},\{\dot{\mathbf{R}}(t)\})\right|$, and c.1-c.4 (right panel): OECD $\mathbf{j}_{e}(x, y, z ;\{\mathbf{R}(t)\},\{\dot{\mathbf{R}}(t)\})$ on $x z$ plane at $y=0$ with four different times $t=0.0 \mathrm{fs}, 3.5 \mathrm{fs}, 4.5 \mathrm{fs}$ and $6.45 \mathrm{fs}$. Horizontal and vertical axes are $x$ and $z$, respectively. Time is expressed in unit of fs. Color codes are indicated at the bottom of each panel in unit of a.u.

cept for the bonding region where a very low elliptical electronic density is observed in Fig. 4a.1. That is, a delocalized electronic density distributed over two nuclei is very sparse in the beginning. From Figs. 4a.2-4a.4, it is recognized that the elliptical electronic density in the bonding region becomes more dense as time is elapsed. In order to understand how many electrons participate to produce the covalent bond, and how many electrons contribute to maintain two independent hydrogen atoms, let us enumerate the following quantities defined as

$$
\begin{aligned}
& n_{s}(\{\mathbf{R}(t)\})=\int d \mathbf{r} \rho_{e}^{s}(\mathbf{r} ;\{\mathbf{R}(t)\}), \\
& n_{c}^{I}(\{\mathbf{R}(t)\})=\int d \mathbf{r} \rho_{e}^{c I}(\mathbf{r} ;\{\mathbf{R}(t)\}) ; \quad I=1,2,
\end{aligned}
$$

where Eqs. (14) and (16) have been used. In Eq. (33) and hereafter, $I=1$ and $I=2$ mean a left and right nuclei, respectively. In Fig. 7a.1, a time dependence of the number of electrons $n_{s}(\{\mathbf{R}(t)\})$ shared by two nuclei is shown. Although $n_{s}(\{\mathbf{R}(t)\}) \cong 0.07$ at the initial time, 


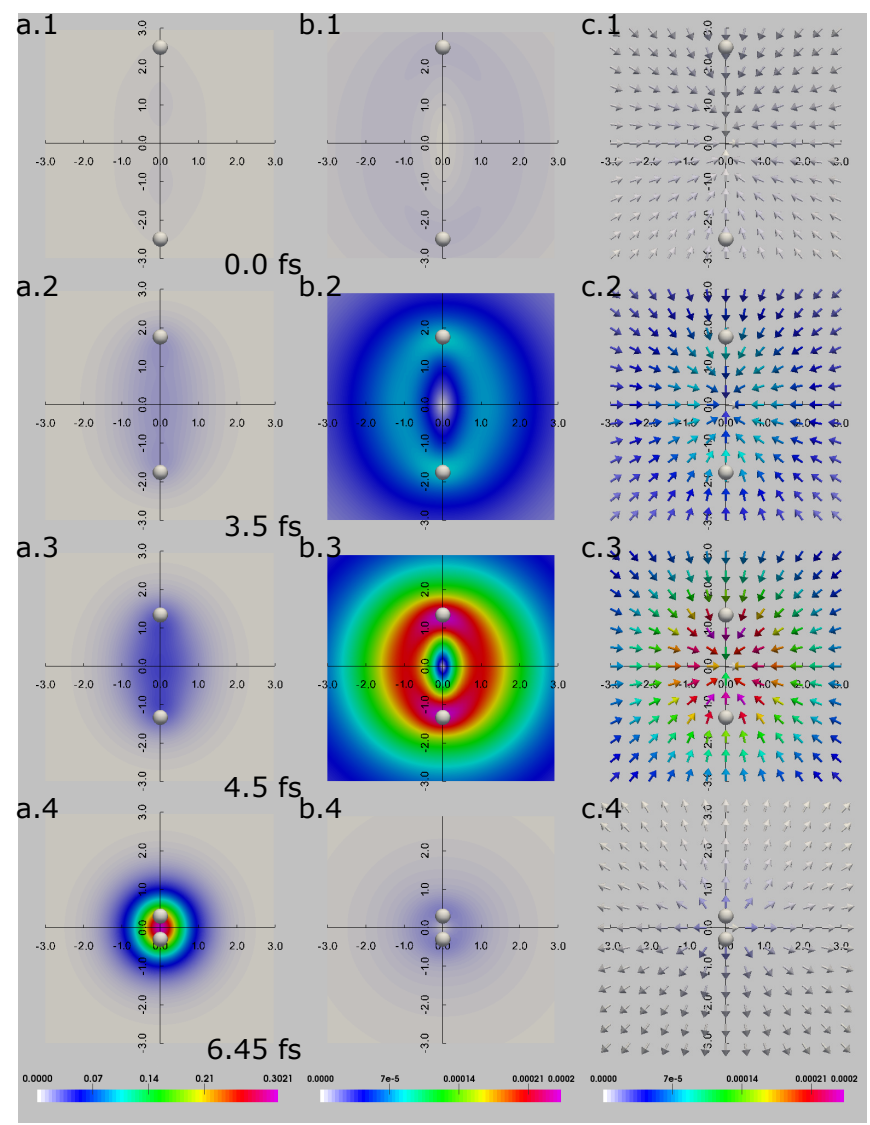

FIG. 4. Color coded contours. a.1-a.4 (left panel): ESD $\rho_{e}^{s}(x, y, z ;\{\mathbf{R}(t)\})$, b.1-b.4 (center panel): absolute value of $\operatorname{ESCD}\left|\mathbf{j}_{e}^{s}(x, y, z ;\{\mathbf{R}(t)\},\{\dot{\mathbf{R}}(t)\})\right|$, and c.1-c.4 (right panel): $\operatorname{ESCD~} \mathbf{j}_{e}^{s}(x, y, z ;\{\mathbf{R}(t)\},\{\dot{\mathbf{R}}(t)\})$. Others are the same as Fig. 3.

it takes $n_{s}(\{\mathbf{R}(t)\}) \cong 0.75$ when $E_{e}(\{\mathbf{R}(t)\})$ takes the minimum value at an internuclear distance 1.341 Bohr [Fig. 6]. That is, 0.75 electrons are shared by two nuclei when the system passes through the minimum point of the potential.

ESCD shown in Figs. 4b.1 and 4c.1 give a very sparse distribution at the initial time in a way consistent with ESD in Fig. 4a.1. As time passes, there appears a very high density region (with an elliptic shell structure) coated with a color between light blue and red in Figs. 4b.2 and 4b.3. This region is surrounded by much lower density region. The farther from this area, the lower the current density. These figures mean that there arises very strong electronic flow around the bonding region, i.e. Region II. From the snapshots of ESCD at $3.5 \mathrm{fs}$ in Fig. 4c.2 and at 4.5 fs in Fig. 4c.3, it is seen that the flow of ESCD is directed toward a center of mass of the hydrogen molecule so as to produce the covalent bond. Namely, Figs. 4b.2 and 4b.3 describes a flow of electronic current toward the bonding region. After passing through the minimum point of $E_{e}(\{\mathbf{R}(t)\})$, ESCD at $6.45 \mathrm{fs}$ is everywhere almost zero as is observed in Figs. 4b. 4 and 4c.4 like the case with OECD in Fig. 3. Due to

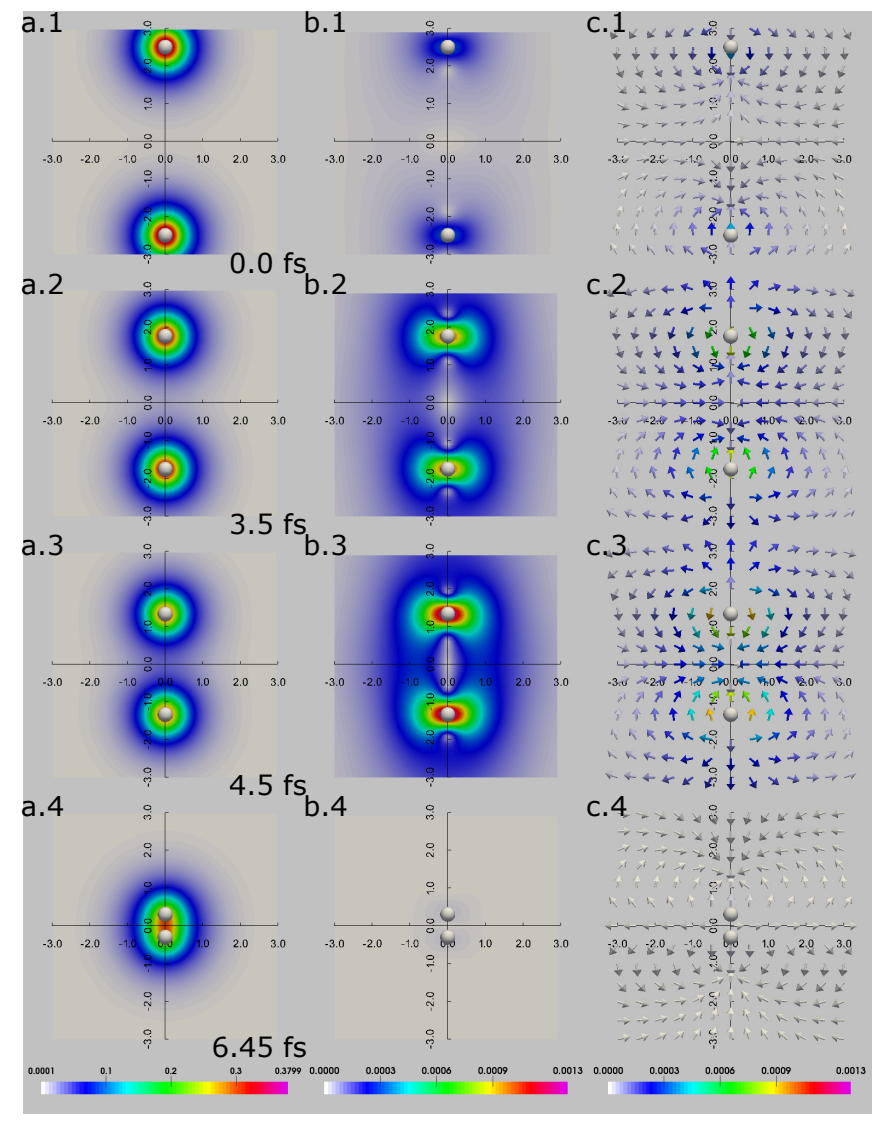

FIG. 5. Color coded contours. a.1-a.4 (left panel): ECD $\rho_{e}^{c}(x, y, z ;\{\mathbf{R}(t)\})$, b.1-b.4 (center panel): absolute value of $\operatorname{ECCD~}\left|\mathbf{j}_{e}^{c}(x, y, z ;\{\mathbf{R}(t)\},\{\mathbf{R}(t)\})\right|$, and c.1-c.4 (right panel): ECCD $\mathbf{j}_{e}^{c}(x, y, z ;\{\mathbf{R}(t)\},\{\dot{\mathbf{R}}(t)\})$. Others are the same as Fig. 3.

the same reason as in the case of OECD discussed previously, the magnitude of ESCD increases until 5.85 fs, then starts to decrease, and finally becomes zero at 6.45 fs.

Snapshots of ECD and ECCD are depicted in Fig. 5. At the initial time, ECD contains two disks, each of which is centered at respective nucleus [Fig. 5a.1]. These two disks approach with each other over time [Figs. 5a.2, 5a.3], and then fuse together [Fig. 5a.4]. These situation are the same as that of OED. In a marked contrast with the case of OED, however, the density of ECD near the center of two disks decreases over time as observed in Figs. 5a.1-5a.3. This decrease is more clearly shown in Fig. 8 where $\rho_{e}^{c I}(\mathbf{r} ;\{\mathbf{R}(t)\})$ defined in Eq. (16) is depicted as a function of distance from the nucleus. From these figures, one may learn how ECD contributing to maintain the structure of hydrogen atoms contracts as time proceeds. This decrease is quantitatively represented by a number of electrons contained in ECD given by $n_{c}^{I}(\{\mathbf{R}(t)\})$. As is shown in Fig. 7a.2, $n_{c}^{I}(\{\mathbf{R}(t)\}) \cong 1.0$ at the initial time, and then monotonically decreases to $n_{c}^{I}(\{\mathbf{R}(t)\}) \cong 0.5$ at $t=6.45$ fs for both $I=1,2$. 


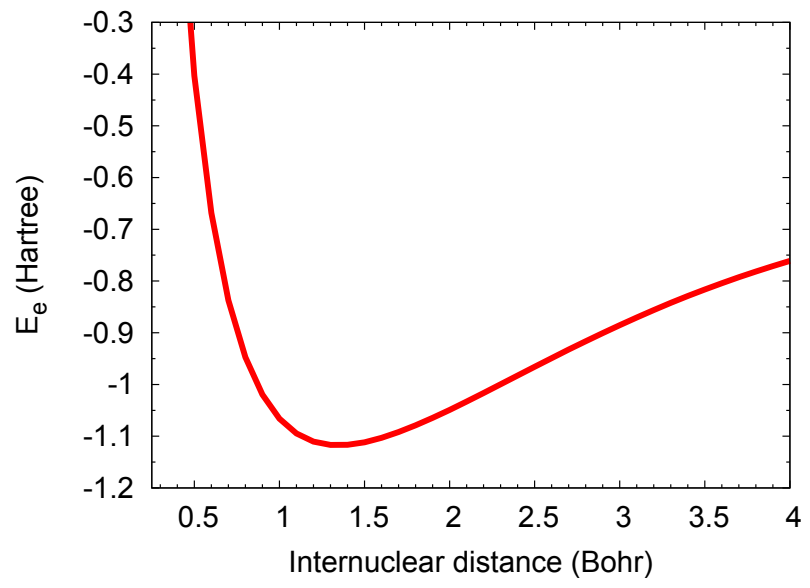

FIG. 6. Adiabatic potential $E_{e}(R)$ as a function of internuclear distance $R$ in units of a.u. Stationary point locates at 1.341 Bohr.

Figures 5b.1-5b.3 shows ECCD which have quite different structure from Fig. 4b. Namely, there appear strong electronic current around two nuclei in Fig. 5b rather than both Region I and Region II (elliptic shell structure) in the case of ESCD. The direction of ECCD in Region I is the same as that of the nuclear motion [Figs. 5c.1-5c.3] and that in Region II is parallel to $x$ axis, and is headed toward the molecular axis [Figs. 5c.2, 5c.3], respectively. Although the results of ECCD looks like the same as that of OECD in Fig. 3, the width of ECCD in Fig. 5b.3 is much smaller than that of OECD in Fig. 3b.3. From the above discussion, a strong electronic current observed in Figs. 5b.2 and 5b.3 is understood to be a flowing out electronic current from around two nuclei in ECD. The magnitude of ECCD shown here increases until $5.8 \mathrm{fs}$, and then changes to decrease, and finally becomes almost zero [Figs. 5b.4, 5c.4] caused by the same reason as that of the OECD.

This subsection is summarized as follows; i) From the initial condition and OED in Fig. 3, the system at the initial time is regarded as two isolated hydrogen atoms in the ground state. ii) As observed from ESD in Fig. 4 and ECD in Fig. 5, the following two phenomena occur simultaneously when the two nuclei get closer; a reduction of electronic density in the vicinity of two nuclei (Region I), and an increase of electronic density in the bonding region. iii) The above simultaneous change is caused by an electronic transfer from Region I to the bonding region, which is visually analyzed through the electronic currents ESCD and ECCD, rather than OECD.

\section{Formation of Covalent Bond}

In the previous subsection, it is shown that, when two hydrogen atoms come close with each other, ESD gets more dense, whereas ECD becomes more sparse than before. It is also discussed that there arises electronic cur-
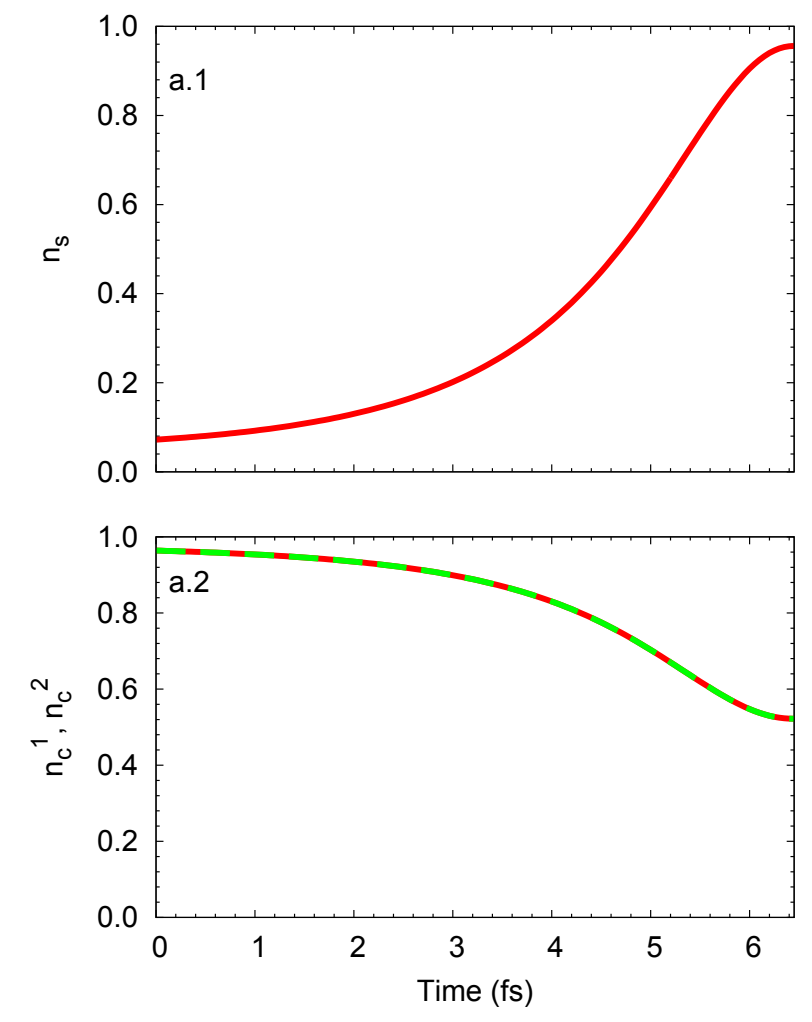

FIG. 7. Upper panel: number of electrons $n_{s}(\{\mathbf{R}(t)\})$ in ESD as a function of time. Lower panel: number of electrons $n_{c}^{1}(\{\mathbf{R}(t)\})$ for nucleus 1 (red dashed line), and $n_{c}^{2}(\{\mathbf{R}(t)\})$ for nucleus 2 (green dashed line) in ECD as a function of time. Time is in units of fs.

rent toward the center of hydrogen molecule (bonding region) from the vicinity of each nucleus (Region I) so as to produce the covalent bond.

In order to understand dynamical reason for producing the hydrogen molecule out of two hydrogen atoms, let us first discuss the atomic internal energy $E^{\text {cont }}(\{\mathbf{R}(t)\})$ given in Eq. (21). By comparing its structure with $\rho_{e}^{c}(\mathbf{r} ;\{\mathbf{R}(t)\})$ in Eqs. (15) and (16), it is clearly recognized that $E^{\text {cont }}(\{\mathbf{R}(t)\})$ sheds light on other aspect of ECD. As shown in Fig. 10a.1, the atomic internal energy at the initial time $E^{\text {cont }}(\{\mathbf{R}(t=0)\}) \cong-0.9$ a.u. which is very close to a twice of the $1 s$ state energy (ionization energy $E_{0}=-0.5$ a.u.) of the hydrogen atom. A quantity defined as $E^{\text {intri }}(\{\mathbf{R}(t)\})=\left(E^{\text {cont }}(\{\mathbf{R}(t)\})-\right.$ $\left.E^{\text {cont }}(\{\mathbf{R}(t=0)\})\right) / 2$ is called an intrinsic excitation energy of hydrogen atoms. $E^{\text {intri }}(\{\mathbf{R}(t)\})$ increases with time and takes the maximum value at $6.45 \mathrm{fs}$. Here, it should be noticed that a number of electrons $n_{c}^{I}(\{\mathbf{R}(t)\})$ introduced in Eq. (33) decreases as a function of time. Taking account of this point, one may understand that a pair of contracted hydrogen atoms is rather highly excited. 


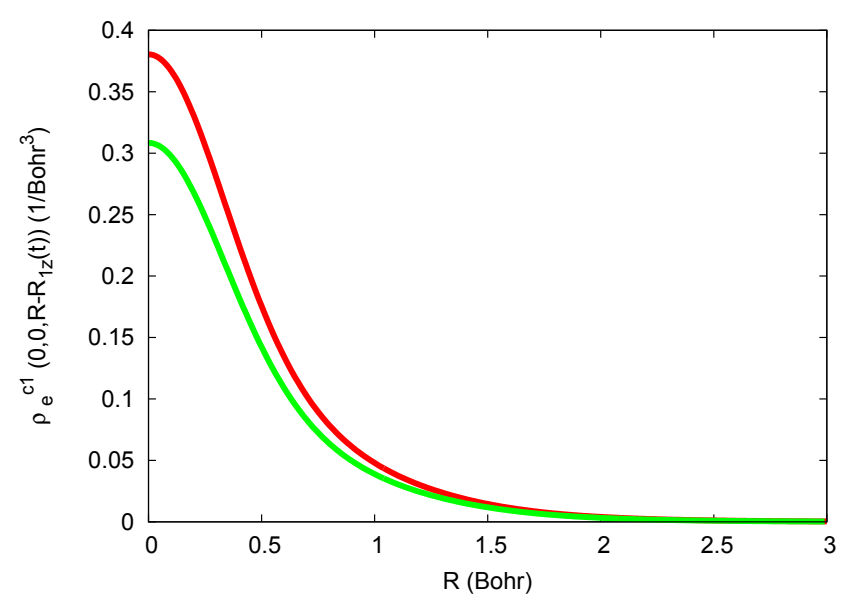

FIG. 8. Contraction of electronic density in hydrogen atom as a function of a distance from the left nucleus. red line: $\rho_{e}^{c 1}\left(0,0, R-R_{1 z}(t) ;\{\mathbf{R}(t)\}\right)$ at $t=0.0 \mathrm{fs}$, green line: $\rho_{e}^{c 1}\left(0,0, R-R_{1 z}(t) ;\{\mathbf{R}(t)\}\right)$ at $t=4.5$ fs. $R_{1 z}(t)$ denotes a $z$ component of a position of the left nucleus at $t$, and $R$ denotes a distance from the left nucleus.

Let us focus on the adiabatic potential $E_{e}(\{\mathbf{R}(t)\})$ which decreases till $5.75 \mathrm{fs}$, and then increases [Fig. 9a.1]. Since the Coulomb force between two nuclei is repulsive, and becomes large when they come close with each other, it is natural to divide $E_{e}(\{\mathbf{R}(t)\})$ into $E_{e}(\{\mathbf{R}(t)\})-V_{n n}(\{\mathbf{R}(t)\})$ and $V_{n n}(\{\mathbf{R}(t)\})$. From Figs. 9a.2 and 9a.3, it is seen that $E_{e}(\{\mathbf{R}(t)\})-V_{n n}(\{\mathbf{R}(t)\})$ decreases, whereas $V_{n n}(\{\mathbf{R}(t)\})$ increases monotonically. Thus, the stationary point of $E_{e}(\{\mathbf{R}(t)\})$ appears as a result of competition between $E_{e}(\{\mathbf{R}(t)\})-V_{n n}(\{\mathbf{R}(t)\})$ and $V_{n n}(\{\mathbf{R}(t)\})$.

Our next task is to examine what term in the righthand side of Eq. (26) causes a decrease of $E_{e}(\{\mathbf{R}(t)\})-$ $V_{n n}(\{\mathbf{R}(t)\})$ which is responsible for making the hydrogen molecule more stable than two separated hydrogen atoms. From Fig. 10a.1, it is apparent that the quantity $E^{\text {cont }}(\{\mathbf{R}(t)\})$ does not contribute to a decrease of $E_{e}(\{\mathbf{R}(t)\})-V_{n n}(\{\mathbf{R}(t)\})$. As is also clear from Fig. 10a.4, the repulsive Coulomb force between electrons $\left\langle\hat{V}_{e e}\right\rangle(\{\mathbf{R}(t)\})$ increases when two nuclei approach with each other. Figure 10a.2 shows that the sharing term of electronic kinetic energy $T_{e}^{\text {shar }}(\{\mathbf{R}(t)\})$ in Eq. (27) increases more rapidly than $\left\langle\hat{V}_{e e}\right\rangle(\{\mathbf{R}(t)\})$. The sharing term $V_{\text {en }}^{\text {shar }}(\{\mathbf{R}(t)\})$ of the attractive Coulomb interaction between electrons and nuclei is depicted as a function of time in Fig 10a.3. From the above numerical results, it is concluded that two hydrogen atoms are combined into one molecule only by the term $V_{e n}^{\text {shar }}(\{\mathbf{R}(t)\})$ alone.

In order to explore which electronic density, either ESD or ECD, plays a dominant role in producing the covalent bond, we divide $V_{e n}^{\text {shar }}(\{\mathbf{R}(t)\})$ into two by using Eqs.
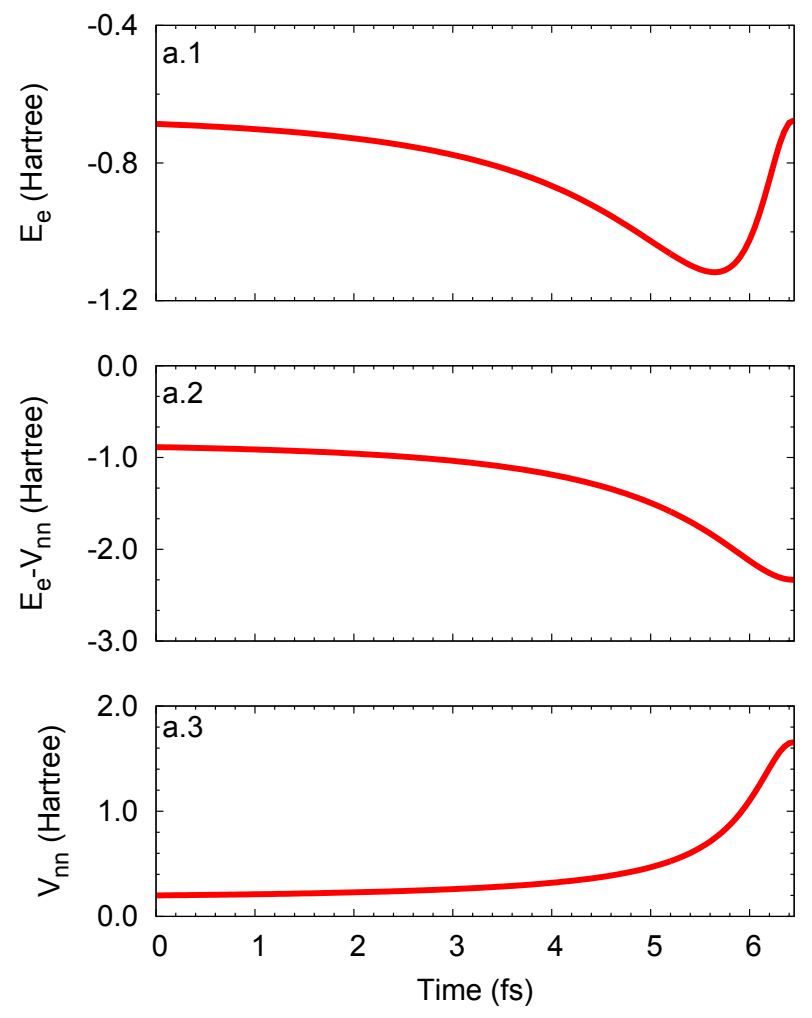

FIG. 9. a.1: Adiabatic potential $E_{e}(\{\mathbf{R}(t)\})$, a.2: $E_{e}(\{\mathbf{R}(t)\})-V_{n n}(\{\mathbf{R}(t)\})$, and a.3: Coulomb energy between nuclei $V_{n n}(\{\mathbf{R}(t)\})$ as a function of time. Time is in units of fs and the others are in units of a.u.

(14) and (16) as

$V_{e n}^{\text {shar }}(\{\mathbf{R}(t)\})=\sum_{I=1}^{N_{n}} v_{e n}^{I}(\{\mathbf{R}(t)\})+\sum_{I(\neq J)=1}^{N_{n}} v_{e n}^{I J}(\{\mathbf{R}(t)\})$,

where

$$
\begin{aligned}
& v_{e n}^{I}(\{\mathbf{R}(t)\})=\int d \mathbf{r} \frac{-1}{\left|\mathbf{r}-\mathbf{R}_{I}(t)\right|} \rho_{e}^{s}(\mathbf{r} ;\{\mathbf{R}(t)\}), \\
& v_{e n}^{I J}(\{\mathbf{R}(t)\})=\int d \mathbf{r} \frac{-1}{\left|\mathbf{r}-\mathbf{R}_{I}(t)\right|} \rho_{e}^{c J}(\mathbf{r} ;\{\mathbf{R}(t)\}) .
\end{aligned}
$$

From the above equation, it is clearly understood that $v_{e n}^{I}(\{\mathbf{R}(t)\})$ expresses the Coulomb energy between the $I$-th nucleus and ESD, and plays a role in stabilizing the covalent bond realized in ESD. On the other hand, $v_{e n}^{I J}(\{\mathbf{R}(t)\})$ expresses the Coulomb energy between the $I$-th nucleus and the electronic density localizing around the $J$-th nucleus. That is $v_{e n}^{I J}(\{\mathbf{R}(t)\})$ tries to draw out an electron from the $J$-th atom, and to put it into the $I$-th nuclei with $I \neq J$. In other words, $v_{e n}^{I J}(\{\mathbf{R}(t)\})$ tries to generate the covalent bond between two atoms ( $I$ and $J$ ), not to leave them as two independent hydrogen atoms. From our numerical calculation depicted in 

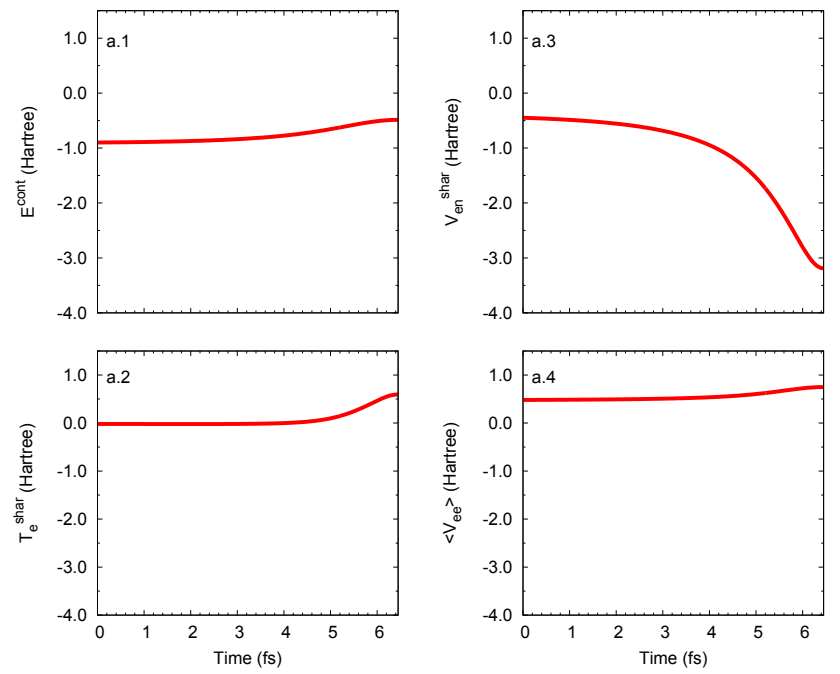

FIG. 10. a.1: Atomic internal energy $E^{\text {cont }}(\{\mathbf{R}(t)\})$, a.2: electronic kinetic energy of sharing term $T_{e}^{\text {shar }}(\{\mathbf{R}(t)\})$, a.3: Coulomb energy between ESD and nuclei $V_{e n}^{\text {shar }}(\{\mathbf{R}(t)\})$, and a.4: Coulomb energy between electrons $\left\langle\hat{V}_{e e}\right\rangle(\{\mathbf{R}(t)\})$ as a function of time. Time is in units of fs and the others are in units of a.u.

Fig. 11, it is seen that both $v_{e n}^{I}(\{\mathbf{R}(t)\})$ and $v_{e n}^{I J}(\{\mathbf{R}(t)\})$ give a negative contribution to $V_{e n}^{\text {shar }}(\{\mathbf{R}(t)\})$, and the former is much more dominated than the latter. From the above discussion, it is clearly understood that the term $V_{e n}^{\text {shar }}(\{\mathbf{R}(t)\})$ plays an essential role in producing the stationary point in the adiabatic potential $E_{e}(\{\mathbf{R}(t)\})$ which conducts two hydrogen atoms to the hydrogen molecule by producing the covalent bond discussed in the previous subsection.

This subsection is summarized as follows; i) When two hydrogen atoms come close with each other, there emerge four effects which prevent two hydrogen atoms from forming the hydrogen molecule; the repulsive Coulomb interactions among electrons $\left\langle\hat{V}_{e e}\right\rangle(\{\mathbf{R}(t)\})$, those among nuclei $V_{n n}(\{\mathbf{R}(t)\})$, a part of electronic kinetic energy $T_{e}^{\text {shar }}(\{\mathbf{R}(t)\})$ and the atomic internal energy $E^{\text {cont }}(\{\mathbf{R}(t)\})$. ii) Paying the excitation energy $E^{i n t r i}(\{\mathbf{R}(t)\})$ to each of hydrogen atoms which try to preserve their atomic structure, $V_{e n}^{\text {shar }}(\{\mathbf{R}(t)\})$ produces the covalent bond by overcoming the repulsive effects coming from $\left\langle\hat{V}_{e e}\right\rangle(\{\mathbf{R}(t)\}), V_{n n}(\{\mathbf{R}(t)\}), T_{e}^{\text {shar }}(\{\mathbf{R}(t)\})$ as well as $V_{n n}(\{\mathbf{R}(t)\})$. iii) When two hydrogen atoms come much closer, $V_{n n}(\{\mathbf{R}(t)\})$ gets much more repulsive energy which can't be overcome by $V_{e n}^{\text {shar }}(\{\mathbf{R}(t)\})$. This competitive process determines a stationary point of the hydrogen molecule.

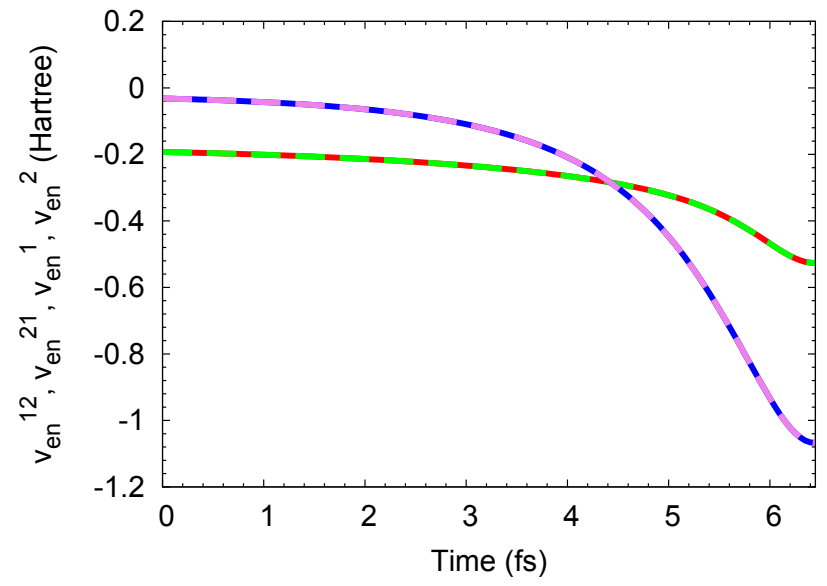

FIG. 11. Each component of $V_{e n}^{\text {shar }}(\{\mathbf{R}(t)\})$ in Eq. (34). Coulomb energy $v_{e n}^{I J}(\{\mathbf{R}(t)\}) ; I=1, J=2$ (red-dashed line), and $I=2, J=1$ (green-dashed line). Coulomb energy $v_{e n}^{I}(\{\mathbf{R}(t)\}) ; I=1$ (blue-dashed line) and $I=2$ (purple-dash line).

\section{SUMMARY}

To explore a dynamical process with respect to the bond and the bond dissociation processes taking place in chemical reactions, it is decisive to define the electronic current density. Since OECD defined directly through the microscopic electronic wave function gives null value under BOMD, it has been considered hard to extract the relevant information from it. Getting over the above difficulty, in this paper, we have proposed to utilize the Maxwell's displacement current density defined by OED under BOMD.

Since OECD thus introduced turned out to be not sufficient in studying the chemical reaction processes, we divided it into two densities; One is composed of the overlap distributions between atomic orbitals belonging to different atoms (sharing density), and is denoted as ESD, whereas the other consists of those belonging to the same atom (contraction density), and is expressed as ECD. ESD plays a role to generate the covalent bond, whereas ECD tries to retain independent character of each atom. In the same way as OED, OECD is divided into ESCD and ECCD. By applying ESCD and ECCD to analize the head-on collision of two hydrogen atoms, in this paper, we have shown how ESD accumulates in the bonding region to create the covalent bond, and how electrons in the ECD go away from each atom by contracting its size.

In order to further explore a direction of reaction process, it is important to understand how the adiabatic potential $E_{e}(\{\mathbf{R}(t)\})$ depends on various parts of OED. By the use of ESD and ECD, it is found that the Coulomb interaction between ESD and nuclei, i.e. $V_{e n}^{\text {shar }}(\{\mathbf{R}(t)\})$ plays a decisive role in producing the covalent bond in the hydrogen molecule. 


\section{ACKNOWLEDGEMENTS}

We are very thankful to N. Inoue (AIST), K. Wen (University of Surrey), M. Fujii (The university of Tokyo), H. Teramoto, T. Yamashita (The university of Tokyo) and Y. Hashimoto (University of Tsukuba) for stimulating discussions and advice.

\section{Appendix A: Calculation of Eq. (11)}

By substituting Eq. (7) to Eq. (11) with Eq. (13), one gets

$$
\begin{aligned}
& \mathbf{j}_{e}(\mathbf{x} ;\{\mathbf{R}(t)\},\{\dot{\mathbf{R}}(t)\})=\frac{1}{4 \pi} \sum_{I J=1}^{N_{n}} \sum_{\mu(I) \nu(J)}^{\mathrm{AO}} \\
& {\left[\nabla_{\mathbf{x}} \phi_{\mu(I) \nu(J)}\left(\mathbf{x} ; \mathbf{R}_{I}(t), \mathbf{R}_{J}(t)\right) \frac{\partial}{\partial t} \rho_{\mu(I) \nu(J)}(\{\mathbf{R}(t)\})\right.} \\
& \left.+\rho_{\mu(I) \nu(J)}(\{\mathbf{R}(t)\}) \frac{\partial}{\partial t} \nabla_{\mathbf{x}} \phi_{\mu(I) \nu(J)}\left(\mathbf{x} ; \mathbf{R}_{I}(t), \mathbf{R}_{J}(t)\right)\right]
\end{aligned}
$$

where

$\phi_{\mu(I) \nu(J)}\left(\mathbf{x} ; \mathbf{R}_{I}(t), \mathbf{R}_{J}(t)\right)=\int d \mathbf{r} \frac{1}{|\mathbf{x}-\mathbf{r}|} \chi_{\mu(I)}(\mathbf{r}) \chi_{\nu(J)}(\mathbf{r})$.

Expanding $\mathrm{AO}$ as a linear combination of the Gaussian type orbitals (GTO) $g_{i}\left(\mathbf{r}-\mathbf{R}_{I}(t)\right)$,

$$
\chi_{\mu(I)}(\mathbf{r})=\sum_{i}^{\mathrm{GTO}} d_{i}^{\mu(I)} g_{i}\left(\mathbf{r}-\mathbf{R}_{I}(t)\right)
$$

one obtains

$$
\begin{aligned}
\phi_{\mu(I) \nu(J)}\left(\mathbf{x} ; \mathbf{R}_{I}(t), \mathbf{R}_{J}(t)\right) & \\
= & \sum_{i j}^{\mathrm{GTO}} d_{i}^{\mu(I)} d_{j}^{\nu(J)} \phi_{i j}\left(\mathbf{x} ; \mathbf{R}_{I}(t), \mathbf{R}_{J}(t)\right)
\end{aligned}
$$

where $d_{i}^{\mu(I)}$ denotes the contraction coefficients and

$$
\begin{aligned}
\phi_{i j}\left(\mathbf{x} ; \mathbf{R}_{I}(t), \mathbf{R}_{J}(t)\right) \\
\quad=\int d \mathbf{r} \frac{1}{|\mathbf{x}-\mathbf{r}|} g_{i}\left(\mathbf{r}-\mathbf{R}_{I}(t)\right) g_{j}\left(\mathbf{r}-\mathbf{R}_{J}(t)\right) .
\end{aligned}
$$

Using the STO-3G basis set, one evaluates $\nabla_{\mathbf{x}} \phi_{i j}\left(\mathbf{x} ; \mathbf{R}_{I}(t), \mathbf{R}_{J}(t)\right)$ and $\partial / \partial t \nabla_{\mathbf{x}} \phi_{i j}\left(\mathbf{x} ; \mathbf{R}_{I}(t), \mathbf{R}_{J}(t)\right)$ of the hydrogen molecule as

$$
\begin{gathered}
\nabla_{\mathbf{x}} \phi_{i j}\left(\mathbf{x} ; \mathbf{R}_{I}(t), \mathbf{R}_{J}(t)\right)=-4 \pi\left(\frac{2 \zeta_{i}}{\pi}\right)^{\frac{3}{2}}\left(\frac{2 \zeta_{j}}{\pi}\right)^{\frac{3}{2}} \\
\times\left(\mathbf{x}-\mathbf{R}_{p}(t)\right) e^{-\frac{\zeta_{i} \zeta_{j}}{\zeta_{p}} R_{I J}^{2}(t)} F_{2}\left(\zeta_{p} x_{p}^{2}(t)\right), \quad(\mathrm{A} 6) \\
\frac{\partial}{\partial t} \nabla_{\mathbf{x}} \phi_{i j}\left(\mathbf{x} ; \mathbf{R}_{I}(t), \mathbf{R}_{J}(t)\right)=4 \pi\left(\frac{2 \zeta_{i}}{\pi}\right)^{\frac{3}{2}}\left(\frac{2 \zeta_{j}}{\pi}\right)^{\frac{3}{2}} e^{-\frac{\zeta_{i} \zeta_{j}}{\zeta_{p}} R_{I J}^{2}(t)} \\
\times\left[\left\{\dot{\mathbf{R}}_{p}(t)+2 \frac{\zeta_{i} \zeta_{j}}{\zeta_{p}}\left(\mathbf{R}_{I}(t)-\mathbf{R}_{J}(t)\right) \cdot\left(\dot{\mathbf{R}}_{I}(t)-\dot{\mathbf{R}}_{J}(t)\right)\left(\mathbf{x}-\mathbf{R}_{p}(t)\right)\right\}\right. \\
\left.\times F_{2}\left(\zeta_{p} x_{p}^{2}(t)\right)-2 p F_{4}\left(\zeta_{p} x_{p}^{2}(t)\right) \dot{\mathbf{R}}_{p}(t) \cdot\left(\mathbf{x}-\mathbf{R}_{p}(t)\right)\left(\mathbf{x}-\mathbf{R}_{p}(t)\right)\right],
\end{gathered}
$$

where $\zeta_{i}$ and $\zeta_{j}$ are orbital coefficients, $\zeta_{p}=\zeta_{i}+\zeta_{j}$, $R_{I J}(t)=\left|\mathbf{R}_{I}(t)-\mathbf{R}_{J}(t)\right|$ and $\mathbf{R}_{p}(t)=\left(\zeta_{i} \mathbf{R}_{I}(t)+\right.$ $\left.\zeta_{j} \mathbf{R}_{J}(t)\right) / \zeta_{p} \cdot F_{n}(x)$ is defined as

$$
F_{n}(x)=\int_{0}^{1} d \tau \tau^{n} e^{-x \tau^{2}}
$$

[1] M. Born and R. Oppenheimer, Ann. Phys. 389, 457 (1927).

[2] H.-D. Meyer and W. H. Miller, J. Chem. Phys. 70, 3214 (1979).

[3] R. Car and M. Parrinello, Phys. Rev. Lett. 55, 2471 (1985).

[4] J. C. Tully and R. K. Preston, J. Chem. Phys. 55, 562 (1971).

[5] U. Manthe, H.-D. Meyer, and L. S. Cederbaum, J. Chem. phys. 97, 3199 (1992).

[6] U. Saalmann and R. Schmidt, Z. Phys. D 38, 153 (1996).

[7] C. Zhu, A. W. Jasper, and D. G. Truhlar, J. Chem. Phys. 120, 5543 (2004).

[8] K. Takatsuka, J. Phys. Chem. A 111, 10196 (2007).

[9] A. Abedi, F. Agostini, and E. Gross, Europhys. Lett. 106, 33001 (2014).
[10] T. Yonehara and S. Kato, J. Chem. Phys. 125, 084307 (2006).

[11] G. Albareda, J. M. Bofill, I. Tavernelli, F. HuarteLarrañaga, F. Illas, and A. Rubio, J. Phys. Chem. Lett. 6, 1529 (2015).

[12] M. Grønager and N. E. Henriksen, J. Phys. Chem. A 102, 4277 (1998).

[13] M. Gro, N. E. Henriksen, et al., J. Chem. Phys. 109, 4335 (1998).

[14] W. R. Duncan, W. M. Stier, and O. V. Prezhdo, J. Am. Chem. Soc. 127, 7941 (2005).

[15] H. Tachikawa, J. Chem. Phys. 125, 144307 (2006).

[16] H. Ushiyama and K. Takatsuka, Angew. Chem. Int. Ed. 46, 587 (2007).

[17] L. Mones, P. J. Rossky, and L. Turi, J. Chem. Phys. 135, 084501 (2011). 
[18] D. Mendive-Tapia, M. Vacher, M. J. Bearpark, and M. A. Robb, J. Chem. Phys. 139, 044110 (2013).

[19] M. Vacher, M. J. Bearpark, and M. A. Robb, J. Chem. Phys. 140, 201102 (2014).

[20] M. Vacher, D. Mendive-Tapia, M. J. Bearpark, and M. A. Robb, J. Chem. Phys. 142, 094105 (2015).

[21] K. Nagashima and K. Takatsuka, J. Phys. Chem. A 113, 15240 (2009).

[22] K. Yamamoto and K. Takatsuka, Chem. Phys. 475, 39 (2016).

[23] L. Landau and E. Lifshitz, Quantum Mechanics, NonRelativistic Theory (Butterworth-Heinemann, 1981).

[24] D. Marx and J. Hutter, Ab initio molecular dynamics: basic theory and advanced methods (Cambridge University Press, 2009).

[25] L. A. Nafie, J. Phys. Chem. A 101, 7826 (1997).

[26] I. Barth, H.-C. Hege, H. Ikeda, A. Kenfack, M. Koppitz, J. Manz, F. Marquardt, and G. K. Paramonov, Chem. Phys. Lett. 481, 118 (2009).

[27] T. Bredtmann, D. J. Diestler, S.-D. Li, J. Manz, J. F. Pérez-Torres, W.-J. Tian, Y.-B. Wu, Y. Yang, and H.-J. Zhai, Phys. Chem. Chem. Phys. 17, 29421 (2015).

[28] M. Okuyama and K. Takatsuka, Chem. Phys. Lett. 476, 109 (2009).

[29] D. J. Diestler, A. Kenfack, J. Manz, and B. Paulus, J. Phys. Chem. A 116, 2736 (2011).

[30] D. J. Diestler, J. Phys. Chem. A 116, 11161 (2012).

[31] G. Hermann, B. Paulus, J. Pérez-Torres, and V. Pohl, Phys. Rev. A 89, 052504 (2014).
[32] S. Patchkovskii, J. Chem. Phys. 137, 084109 (2012).

[33] T. Sun and R. M. Wentzcovitch, Chem. Phys. Lett. 554, 15 (2012).

[34] D. Diestler, J. Phys. Chem. A 117, 4698 (2013).

[35] V. Pohl and J. C. Tremblay, Phys. Rev. A 93, 012504 (2016).

[36] A. Schild, F. Agostini, and E. K. Gross, J. Phys. Chem. A 120, 3316 (2016).

[37] K. Ruedenberg, Rev. Mod. Phys. 34, 326 (1962).

[38] W. Heitler and F. London, Z. Phys 44, 455 (1927).

[39] H. Hellmann, Z. Phys 85, 180 (1933).

[40] J. C. Slater, J. Chem. Phys. 1, 687 (1933).

[41] C. W. Wilson Jr and W. A. Goddard III, Theor. Chem. Acc. 26, 195 (1972).

[42] W. Kutzelnigg, Angew. Chem. Int. Ed. 12, 546 (1973).

[43] W. Kutzelnigg and W. Schwarz, Phys. Rev. A 26, 2361 (1982).

[44] K. Ruedenberg and M. W. Schmidt, J. Comput. Chem. 28, 391 (2007).

[45] G. B. Bacskay and S. Nordholm, J. Phys. Chem. A 117, 7946 (2013).

[46] I. Mayer, J. Phys. Chem. A 118, 2543 (2014).

[47] C. C. J. Roothaan, Rev. Mod. Phys. 23, 69 (1951).

[48] R. P. Feynman, Phys. Rev. 56, 340 (1939).

[49] J. D. Jackson, Classical electrodynamics (Wiley, 1999).

[50] M. W. Schmidt, K. K. Baldridge, J. A. Boatz, S. T. Elbert, M. S. Gordon, J. H. Jensen, S. Koseki, N. Matsunaga, K. A. Nguyen, S. Su, et al., J. Comput. Chem. 14, 1347 (1993). 